\title{
Dexamethasone levels predict cortisol response after infant cardiopulmonary bypass
}

\author{
Sheri S. Crow, MD, ${ }^{a}$ William C. Oliver, Jr, MD, ${ }^{b}$ Jamie A. Kiefer, PA, ${ }^{c}$ Melissa R. Snyder, PhD, ${ }^{d}$ \\ Joseph A. Dearani, MD, ${ }^{c}$ Zhuo Li, MS, ${ }^{e}$ and Harold M. Burkhart, MD $^{c}$
}

\begin{abstract}
Objectives: We sought to evaluate whether there is variability in blood dexamethasone levels after a standard $1 \mathrm{mg} / \mathrm{kg}$ dose of dexamethasone administered before infant cardiopulmonary bypass. We hypothesized that postoperative dexamethasone drug levels are highly variable, and that the infant stress response is related inversely to the amount of dexamethasone measured in the blood.
\end{abstract}

Methods: Thirty-two infants (age, $\leq 365$ days) received $1 \mathrm{mg} / \mathrm{kg}$ of dexamethasone before cardiopulmonary bypass (CPB) initiation. Blood was analyzed for cortisol, adrenocorticotropin, and interleukin (IL)-6, IL-8, and IL-10 levels after anesthesia induction, after CPB, after intensive care unit (ICU) arrival, and 4, 8, 12, and 24 hours after surgery. Patients were grouped as high dexamethasone $(\geq 15 \mu \mathrm{g} / \mathrm{dL})$ or low dexamethasone $(<15 \mu \mathrm{g} / \mathrm{dL})$ based on their level at ICU arrival.

Results: Dexamethasone levels varied significantly between the high $(\mathrm{n}=22)$ and low $(\mathrm{n}=10)$ dexamethasone groups throughout the entire postoperative course and were correlated highly with cortisol response. Patients with high dexamethasone levels had postoperative cortisol levels that were lower than their pre-CPB baseline cortisol levels. Cortisol levels remained low throughout the first 24 postoperative hours even after dexamethasone levels neared zero. There were no significant differences between groups in the duration of mechanical ventilation or ICU length of stay.

Conclusions: Dexamethasone levels are highly variable at ICU arrival, despite standardized $1 \mathrm{mg} / \mathrm{kg}$ dosing before CPB initiation. (J Thorac Cardiovasc Surg 2014;147:475-82)

Corticosteroids remain a controversial but widely used strategy for mediating the inflammatory/stress response after infant cardiopulmonary bypass (CPB). ${ }^{1}$ Despite decades of research investigating this question, the optimal indication, drug type, dose, and timing for perioperative corticosteroid administration remains unclear. As a result, the approach to corticosteroid administration varies significantly within and between cardiac surgical programs. ${ }^{2}$ Surveys show that the younger the patient, the more likely they are to receive pre-bypass corticosteroids at a majority of US cardiac surgical centers. ${ }^{2}$ Infants represent a subset of congenital heart disease (CHD) patients who frequently present with complex CHD and require surgical intervention early in life to optimize survival. These procedures continue to have the highest

\footnotetext{
From the Division of Pediatric Critical Care, ${ }^{a}$ Department of Pediatrics and Adolescent Medicine, Department of Anesthesiology, ${ }^{\mathrm{b}}$ Division of Cardiothoracic Surgery, ${ }^{\mathrm{c}}$ Department of Laboratory Medicine, ${ }^{\mathrm{d}}$ and Division of Statistics, ${ }^{\mathrm{e}}$ Mayo Clinic, Rochester, Minn.

Disclosures: Authors have nothing to disclose with regard to commercial support.

Read at the 93rd Annual Meeting of The American Association for Thoracic Surgery, Minneapolis, Minnesota, May 4-8, 2013.

Received for publication May 4, 2013; revisions received Aug 26, 2013; accepted for publication Sept 4, 2013; available ahead of print Nov 11, 2013.

Address for reprints: Sheri S. Crow, MD, Division of Pediatric Critical Care, Department of Pediatrics and Adolescent Medicine, Mayo Clinic, 200 First St SW, Rochester, MN 55905 (E-mail: Crow.sheri@mayo.edu).

$0022-5223 / \$ 36.00$

Copyright (c) 2014 by The American Association for Thoracic Surgery

http://dx.doi.org/10.1016/j.jtcvs.2013.09.023
}

risk for surgical mortality and require longer CPB times. The heightened infant systemic inflammatory response to CPB can lead to postoperative cardiac dysfunction and hemodynamic lability. ${ }^{3,4}$ Pre-CPB corticosteroid administration in this setting is believed to improve hemodynamic stability, reduce fluid and vasopressor/inotrope requirements, and provide cardiac myocyte protection after CPB. ${ }^{1,5,6}$

Many pediatric studies have attempted to advance our understanding of the optimal corticosteroid approach for mediating the infant perioperative cardiac surgical stress response. Unfortunately, variability in study designs, clinical practice, and patient selection has limited the ability of these investigations to inform clinicians regarding best practices for perioperative corticosteroid administration. In general, these studies have generated more questions than answers. Achieving consensus surrounding corticosteroid therapy requires a more detailed understanding of how corticosteroids interact with the innate stress response. We know that corticosteroid administration inhibits the patient's adrenal glands from releasing cortisol through a negative feedback loop. ${ }^{7}$ However, the magnitude and duration of this corticosteroid-induced suppression after CPB is not well defined. Pharmacokinetic evaluations of methylprednisolone and $\mathrm{CPB}$ would suggest that a standard weight-based corticosteroid dose does not translate consistently into equivalent drug levels in the blood for every patient. $^{8}$ Patient pharmacokinetics, hemodilution, 


$$
\begin{aligned}
& \text { Abbreviations and Acronyms } \\
& \begin{aligned}
\text { ACTH } & =\text { adrenocorticotropin } \\
\mathrm{CHD} & =\text { congenital heart disease } \\
\mathrm{CPB} & =\text { cardiopulmonary bypass } \\
\mathrm{HCT} & =\text { hematocrit } \\
\mathrm{ICU} & =\text { intensive care unit } \\
\mathrm{IL} & =\text { interleukin } \\
\text { RACHES } & =\text { Risk Adjustment for Congenital Heart } \\
& \text { Surgery }
\end{aligned}
\end{aligned}
$$

volume of modified ultrafiltration, and transfusion have the potential to impact postoperative corticosteroid drug levels and lead to variability in corticosteroid suppression of the innate cortisol response. Past investigations seeking to correlate cortisol levels with clinical outcomes have not characterized this potential interaction between corticosteroid drug levels and the patient cortisol response.

We sought to evaluate whether there is variability in blood dexamethasone levels after a standard $1 \mathrm{mg} / \mathrm{kg}$ dose of dexamethasone administered before infant cardiopulmonary bypass. We hypothesized that postoperative dexamethasone drug levels are highly variable, and that the infant stress response is related inversely to the amount of dexamethasone measured in the blood. To test this theory we simultaneously measured blood levels of dexamethasone and cortisol at critical time points during the perioperative period for infants undergoing CPB for CHD surgery.

\section{MATERIALS AND METHODS \\ Study Design and Patient Selection}

We conducted a prospective trial of 32 infants (age, $\leq 365$ days) who presented to a single institution for congenital cardiac surgery from March 2009 to September 2010. Eligible participants were all infants who would require $\mathrm{CPB}$ during their surgical procedure. Exclusion criteria included prematurity (gestational age, $<36$ weeks), corticosteroid administration within 24 hours preceding surgery, and preoperative mechanical circulatory support. This study was approved by the Institutional Review Board of the Mayo Clinic and parents provided signed informed consent.

\section{Study Procedures and End Points}

Blood was collected from study participants at 7 time points: (1) after anesthesia induction, (2) after premodified ultrafiltration, (3) intensive care unit (ICU) arrival, (4) postoperative hour 4, (5) postoperative hour 8, (6) postoperative hour 12, and (7) postoperative hour 24. At each of the 7 time points, blood was analyzed for the following levels: dexamethasone, cortisol, adrenocorticotropin (ACTH), interleukin (IL)-6, IL-8, and IL-10.

\section{Laboratory Analysis}

ACTH level was measured using a solid-phase, sequential immunoassay on the Siemens Immulite 2000 automated immunoassay system (Siemens Healthcare Diagnostics, Deerfield, Ill). Dexamethasone and cortisol were measured by liquid chromatography-tandem mass spectrometry (ThermoFisher Scientific, Franklin, Mass, and Applied Biosystems-MDS Sciex, Foster City, Calif).

The IL-6, IL-8, and IL-10 assays were completed using a 20-plex Procarta Cytokine Kit (Affymetrix, Santa Clara, Calif). All assays were performed on a BioPlex 200 using BioPlex Manager 5.0 software
(Bio-Rad, Hercules, Calif). Lyophilized standards and controls also were obtained from Affymetrix. Standards were hydrated in a plasma matrix and serially diluted over a measuring range of 0.56 to $1215 \mathrm{pg} / \mathrm{mL}$. Controls were hydrated in the same matrix at concentrations of 175.0 and $12.0 \mathrm{pg} / \mathrm{mL}$. All plasma ethylenediaminetetraacetic acid samples were run in duplicate, at a minimum. Results were analyzed with the BioPlex 5.0 software package (Bio-Rad) using a 5-parameter logistics curve based on 8 standard points.

\section{Corticosteroid Administration}

Patients were treated according to the Mayo Clinic's standard corticosteroid protocol. Each infant received a $0.5 \mathrm{mg} / \mathrm{kg}$ dose of dexamethasone at anesthesia induction and CPB initiation to equal a total intraoperative dexamethasone dose of $1 \mathrm{mg} / \mathrm{kg}$.

\section{Anesthesia Induction and Cardiopulmonary Bypass Management}

Anesthesia induction was achieved with fentanyl $(25-50 \mu \mathrm{g} / \mathrm{kg})$; midazolam $(0.1 \mathrm{mg} / \mathrm{kg})$, a muscle relaxant; and titration of a volatile anesthetic agent. The CPB extracorporeal circuit was primed with Plasmalyte (Baxter Healthcare Corp, Deerfield, Ill), 10 to $20 \mathrm{mEq}$ sodium bicarbonate $8.5 \%, 0.5 \mathrm{~g} / \mathrm{kg}$ mannitol $12.5 \%$, and $100 \mathrm{~mL}$ of $25 \%$ albumin. No corticosteroids were given in the pump prime. Washed packed red blood cells were added to the circuit prime to achieve a hematocrit (HCT) of 25 to 30 upon initiation of CPB. In addition, fresh-frozen plasma was added to the pump prime in all patients weighing less than $5 \mathrm{~kg}$ and on select patients weighing between 5 and 10 $\mathrm{kg}$. If the temperature was to remain above $32^{\circ} \mathrm{C}$, the HCT was kept above $25 \%$ and an alpha-stat oxygenator ventilation regimen was used. Nonpulsatile flow during CPB was maintained at 2.2 to $2.6 \mathrm{~L} / \mathrm{min} / \mathrm{m}^{2}$. Patient temperature was monitored by a nasopharyngeal and urinary catheter probe for patients weighing more than $4 \mathrm{~kg}$ and only by nasopharyngeal probe for patients weighing less than $4 \mathrm{~kg}$. For deep hypothermia procedures with circulatory arrest or low-flow perfusion, a $\mathrm{pH}$-stat cooling regimen was used during temperatures lower than $32^{\circ} \mathrm{C}$ and alpha-stat management was used upon rewarming. Myocardial protection was accomplished with 20 to $30 \mathrm{~mL} / \mathrm{kg}$ of highpotassium cardioplegic solution, with additional cardioplegia given as needed in a 10 to $15 \mathrm{~mL} / \mathrm{kg}$ dose using a 1:4 blood crystalloid cardioplegic solution. Upon rewarming, the HCT was maintained at greater than $24 \%$ to $30 \%$. All patients received conventional ultrafiltration during $\mathrm{CPB}$. Arteriovenous modified ultrafiltration was performed for 15 to 20 minutes after separation from CPB in all patients. All patients were admitted postoperatively to a dedicated congenital cardiac intensive care unit and managed by a cardiac intensivist.

\section{Patient Comparisons and Outcome Variables}

Dexamethasone blood levels were compared at ICU arrival (time 3) and subjects were classified as high dexamethasone (dexamethasone level, $\geq 15 \mu \mathrm{g} / \mathrm{dL}$ ) and low dexamethasone (dexamethasone level, $<15 \mu \mathrm{g} / \mathrm{dL}$ ). The primary outcome measure compared between the high and low dexamethasone groups was cortisol level at times 3, 4, and 5. Secondary outcome measures included the duration of mechanical ventilation, ICU length of stay, ACTH levels, IL-6, IL-8, and IL-10 levels.

\section{Statistical Analysis}

Descriptive statistics for categoric variables are reported as frequencies and percentages and continuous variables are reported as means \pm standard deviations with medians. Continuous variables measuring drug and blood levels at different time points were compared between high dexamethasone and low dexamethasone groups using 2-sample $t$ test or Wilcoxon rank sum test where appropriate. All statistical tests were 2 sided, with the $\alpha$ level set at 0.05 for statistical significance.

\section{RESULTS}

A total of 32 infants undergoing cardiac surgery with CPB had blood samples collected and analyzed for 


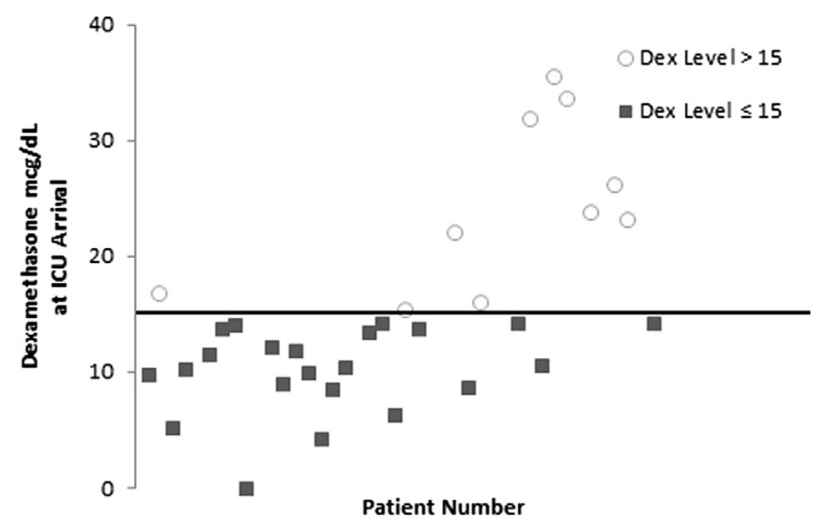

FIGURE 1. Distribution of dexamethasone levels at ICU arrival. A scatterplot of the dexamethasone level for each patient as measured at ICU arrival. The black line denotes a dexamethasone level of 15. Based on the scatterplot distribution, patients were classified into 2 groups: high dexamethasone level at ICU arrival of 15 or higher $(n=10)$ and low dexamethasone level at ICU arrival of less than $15(\mathrm{n}=22)$. $I C U$, Intensive care unit; Dex, dexamethasone.

dexamethasone, cortisol, and ACTH levels at 7 time points during their perioperative course.

Dexamethasone drug levels measured in the blood at ICU arrival were highly variable despite the standard $1 \mathrm{mg} / \mathrm{kg}$ dose of dexamethasone administered to all patients before CPB initiation. The dexamethasone level for each patient at ICU arrival (time 3) was used to categorize the patients into 2 groups: high dexamethasone (dexamethasone level, $\geq 15 \mu \mathrm{g} / \mathrm{dL}$ ) versus low dexamethasone (dexamethasone level, $\leq 15 \mu \mathrm{g} / \mathrm{dL}$ ) to facilitate comparison of dexamethasone level with outcome measures. A dexamethasone level of 15 was chosen as the cut-off point, based on the observation that the majority of patients had dexamethasone levels above or below this level, generating a natural dividing line through the cohort (Figure 1). Ten patients met the criteria for high dexamethasone and 22 patients met the criteria for low dexamethasone, and these levels were significantly different between the 2 groups at each time point measured (Figure 2).

Patient characteristics are shown in Table 1. There were no significant differences between groups with regard to age, sex, CPB and aortic cross-clamp time, Risk Adjustment for Congenital Heart Surgery (RACHS-1) category, or classification of $\mathrm{CHD}$ as cyanotic or single-ventricle physiology. Patients with high dexamethasone levels trended toward lower inotrope scores at 24 hours but this difference was not significant. Fifteen patients received an additional dose of dexamethasone before extubation. These patients were censored from the analysis at the time they received these postoperative steroids to prevent confounding of the dexamethasone, cortisol, ACTH, and IL level measurements.

Both groups had similar cortisol levels at anesthesia induction (predexamethasone) that decreased sharply
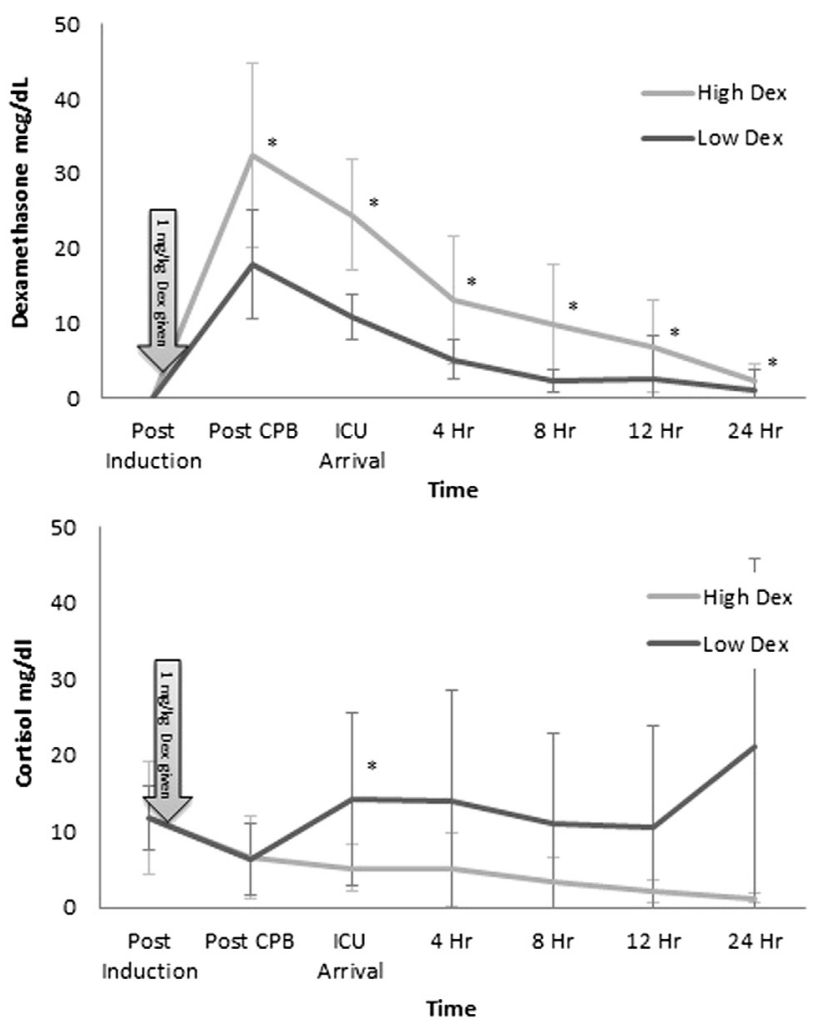

FIGURE 2. Dexamethasone and cortisol levels for the high dexamethasone versus low dexamethasone groups. Top: Mean dexamethasone levels at each time point. Bottom: Mean cortisol responses over time for patients with high $(>15)$ versus low $(\leq 15)$ dexamethasone levels. *Significant differences between groups at the corresponding time point. $I C U$, Intensive care unit; $C P B$, cardiopulmonary bypass; Dex, dexamethasone.

after dexamethasone administration (time 2, end of CPB). Cortisol levels diverged significantly at ICU arrival between the high and low dexamethasone groups (Figure 2). Patients in the high dexamethasone group had lower cortisol levels $(<5)$ throughout the postoperative period. In fact, cortisol levels measured during the first 24 postoperative hours were lower than the baseline/pre-CPB cortisol levels and remained low even after drug levels of dexamethasone had decreased to near zero (Table 2). In contrast, the low dexamethasone group showed an increase in cortisol at ICU arrival compared with baseline/pre-CPB levels. These levels climbed and peaked within 4 hours (time 4) after surgery before returning to the pre- $\mathrm{CPB} / \mathrm{b}$ aseline levels. ACTH levels followed a similar pattern to the cortisol levels for each group. Patients with high dexamethasone levels had a suppressed ACTH response throughout the entire postoperative course (Table 2).

The fluctuations in perioperative cytokine responses were very similar between the high and low dexamethasone groups. However, the low dexamethasone group showed a greater postoperative increase in the proinflammatory 
TABLE 1. Patient characteristics

\begin{tabular}{|c|c|c|c|}
\hline Characteristics & $\begin{array}{c}\text { Low } \\
\text { dexamethasone } \\
(\mathbf{n}=\mathbf{2 2})\end{array}$ & $\begin{array}{c}\text { High } \\
\text { dexamethasone } \\
(\mathbf{n}=10)\end{array}$ & $\begin{array}{r}P \\
\text { valu }\end{array}$ \\
\hline \multicolumn{4}{|l|}{ Demographic variables } \\
\hline Age (d) & $199 \pm 114$ & $225 \pm 363$ & .83 \\
\hline Male, $n(\%)$ & $10(45 \%)$ & $3(30 \%)$ & .66 \\
\hline \multicolumn{4}{|l|}{ RACHS-1 score } \\
\hline $1-3, \mathrm{n}$ & 18 & 9 & .9 \\
\hline $4-6, n$ & 4 & 1 & \\
\hline $\begin{array}{l}\text { Cyanotic congenital } \\
\text { heart disease, } \mathrm{n}(\%)\end{array}$ & $15(68 \%)$ & $4(40 \%)$ & .22 ra \\
\hline $\begin{array}{l}\text { Single ventricle } \\
\text { physiology, n (\%) }\end{array}$ & $3(30 \%)$ & $3(14 \%)$ & .5 \\
\hline CPB time (min) & $104 \pm 44$ & $90 \pm 35$ & .35 \\
\hline Cross-clamp time (min) & $51 \pm 32$ & $45 \pm 28$ & .63 \\
\hline Modified ultrafiltration (mL) & $365 \pm 192$ & $293 \pm 295$ & .4 \\
\hline \multicolumn{4}{|l|}{ Inotrope score } \\
\hline $12 \mathrm{~h}$ & 7.4 & 5.1 & .12 \\
\hline $24 \mathrm{~h}$ & 7.2 & 4.6 & .0 \\
\hline $\begin{array}{l}\text { Mechanical ventilation } \\
\text { duration (d) }\end{array}$ & $3.9 \pm 8.0$ & $1.6 \pm 1.4$ & 1.00 \\
\hline ICU length of stay (d) & $12 \pm 14$ & $11 \pm 11$ & .7 \\
\hline
\end{tabular}

Data are reported as mean \pm standard deviation. RACHS-1, Risk Adjustment for Congenital Heart Surgery; $I C U$, intensive care unit; $C P B$, cardiopulmonary bypass.

cytokines IL-6 and IL-8. There was no significant difference in the postoperative anti-inflammatory IL-10 levels between groups (Table 3).

TABLE 2. Patient results for times 1 through 7

\begin{tabular}{|c|c|c|c|c|c|}
\hline Time periods & $\mathbf{n}$ & $\begin{array}{c}\text { Low } \\
\text { dexamethasone }\end{array}$ & $\mathbf{n}$ & $\begin{array}{c}\text { High } \\
\text { dexamethasone }\end{array}$ & $\begin{array}{c}P \\
\text { value }\end{array}$ \\
\hline \multicolumn{6}{|l|}{ Dexamethasone } \\
\hline 1 & 22 & $0.00 \pm 0.00$ & 10 & $0.00 \pm 0.00$ & .980 \\
\hline 2 & 22 & $17.92 \pm 7.27$ & 10 & $32.48 \pm 12.35$ & .005 \\
\hline 3 & 21 & $10.88 \pm 3.03$ & 10 & $24.48 \pm 7.37$ & $<.001$ \\
\hline 4 & 22 & $5.17 \pm 2.71$ & 9 & $13.18 \pm 8.51$ & .002 \\
\hline 5 & 17 & $2.25 \pm 1.43$ & 8 & $9.90 \pm 7.98$ & .006 \\
\hline 6 & 17 & $2.44 \pm 5.91$ & 6 & $6.85 \pm 6.19$ & .009 \\
\hline 7 & 11 & $1.05 \pm 2.78$ & 5 & $2.31 \pm 2.27$ & .023 \\
\hline \multicolumn{6}{|l|}{ Cortisol } \\
\hline 1 & 22 & $11.70 \pm 4.24$ & 10 & $11.77 \pm 7.36$ & .978 \\
\hline 2 & 22 & $6.35 \pm 4.65$ & 10 & $6.58 \pm 5.35$ & .887 \\
\hline 3 & 22 & $14.17 \pm 11.33$ & 10 & $5.19 \pm 3.03$ & .016 \\
\hline 4 & 22 & $14.07 \pm 14.52$ & 9 & $5.07 \pm 4.79$ & .078 \\
\hline 5 & 17 & $11.00 \pm 11.81$ & 8 & $3.30 \pm 3.34$ & .180 \\
\hline 6 & 17 & $10.42 \pm 13.45$ & 6 & $2.08 \pm 1.54$ & .172 \\
\hline 7 & 12 & $21.07 \pm 24.74$ & 5 & $1.18 \pm 0.58$ & .018 \\
\hline \multicolumn{6}{|l|}{ ACTH } \\
\hline 1 & 21 & $25.62 \pm 15.86$ & 10 & $28.20 \pm 15.63$ & .674 \\
\hline 2 & 22 & $12.48 \pm 13.06$ & 10 & $13.13 \pm 17.57$ & .918 \\
\hline 3 & 22 & $37.66 \pm 68.27$ & 10 & $10.62 \pm 6.76$ & .080 \\
\hline 4 & 22 & $16.25 \pm 17.84$ & 9 & $6.79 \pm 3.12$ & .025 \\
\hline 5 & 17 & $14.43 \pm 22.93$ & 8 & $5.29 \pm 2.43$ & .123 \\
\hline 6 & 17 & $10.92 \pm 14.09$ & 6 & $5.80 \pm 2.97$ & .174 \\
\hline 7 & 10 & $22.19 \pm 23.52$ & 5 & $6.42 \pm 4.82$ & .069 \\
\hline
\end{tabular}

ACTH, Adrenocorticotropin.
TABLE 3. Cytokine responses for times 1 though 7

\begin{tabular}{|c|c|c|c|c|c|}
\hline Time periods & $\mathbf{n}$ & $\begin{array}{c}\text { Low } \\
\text { dexamethasone } \\
\end{array}$ & $\mathbf{n}$ & $\begin{array}{c}\text { High } \\
\text { dexamethasone } \\
\end{array}$ & $\begin{array}{c}P \\
\text { value }\end{array}$ \\
\hline \multicolumn{6}{|l|}{ IL-6 } \\
\hline 1 & 15 & $2.46 \pm 1.53$ & 10 & $2.32 \pm 0.82$ & .697 \\
\hline 2 & 15 & $19.15 \pm 32.76$ & 10 & $9.20 \pm 9.69$ & .305 \\
\hline 3 & 15 & $26.38 \pm 16.03$ & 10 & $19.27 \pm 13.64$ & .256 \\
\hline 4 & 15 & $21.65 \pm 9.95$ & 9 & $14.87 \pm 11.61$ & .043 \\
\hline 5 & 12 & $22.13 \pm 10.64$ & 8 & $18.66 \pm 11.12$ & .335 \\
\hline 6 & 12 & $22.12 \pm 10.39$ & 6 & $13.99 \pm 6.37$ & .059 \\
\hline 7 & 9 & $23.46 \pm 10.34$ & 5 & $13.82 \pm 9.87$ & .116 \\
\hline \multicolumn{6}{|l|}{ IL-8 } \\
\hline 1 & 15 & $1.33 \pm 1.72$ & 10 & $1.30 \pm 0.62$ & .127 \\
\hline 2 & 15 & $5.40 \pm 4.91$ & 10 & $5.93 \pm 6.59$ & .890 \\
\hline 3 & 15 & $7.79 \pm 5.11$ & 10 & $6.74 \pm 4.80$ & .637 \\
\hline 4 & 15 & $5.69 \pm 4.24$ & 9 & $5.12 \pm 3.10$ & 1.000 \\
\hline 5 & 12 & $5.43 \pm 3.69$ & 8 & $4.41 \pm 2.35$ & .758 \\
\hline 6 & 12 & $5.41 \pm 3.70$ & 6 & $3.23 \pm 1.51$ & .098 \\
\hline 7 & 9 & $7.57 \pm 7.49$ & 5 & $2.96 \pm 1.05$ & .240 \\
\hline \multicolumn{6}{|l|}{ IL-10 } \\
\hline 1 & 15 & $1.58 \pm 3.61$ & 10 & $0.95 \pm 0.78$ & .518 \\
\hline 2 & 15 & $54.76 \pm 40.46$ & 10 & $40.11 \pm 27.95$ & .296 \\
\hline 3 & 15 & $46.64 \pm 27.87$ & 10 & $44.77 \pm 32.13$ & .879 \\
\hline 4 & 15 & $11.96 \pm 6.41$ & 9 & $7.14 \pm 10.29$ & .015 \\
\hline 5 & 12 & $7.46 \pm 6.23$ & 8 & $3.77 \pm 2.22$ & .097 \\
\hline 6 & 12 & $5.64 \pm 5.58$ & 6 & $2.51 \pm 0.67$ & .174 \\
\hline 7 & 9 & $13.87 \pm 30.87$ & 5 & $1.78 \pm 1.49$ & .518 \\
\hline
\end{tabular}

\section{DISCUSSION}

We showed that a standardized $1 \mathrm{mg} / \mathrm{kg}$ dose of dexamethasone administered before $\mathrm{CPB}$, results in postoperative dexamethasone blood levels that are highly variable. Infants could be divided into high and low dexamethasone responders based on their drug levels at ICU arrival. These levels were highly correlated with the amount of cortisol suppression observed during the first 24 postoperative hours. Patients with higher postoperative blood levels of dexamethasone showed lower cortisol levels throughout the entire postoperative period. For these patients, cortisol suppression persisted even after dexamethasone levels were near zero. The variations in cortisol response observed between groups (high dexamethasone vs low dexamethasone) was not accounted for by differences in CPB time, surgical case complexity, aortic cross-clamp time, modified ultrafiltration volume, type of congenital heart disease, or the magnitude of the inflammatory response.

Studies investigating the infant post-CPB stress response have been unable to identify consistent correlations between cortisol level and clinical outcomes to define an appropriate cortisol/stress response. This knowledge gap remains at the heart of the perioperative corticosteroid debate and hinders the design and interpretation of past and future research investigations. The inconsistency and 
wide ranges observed in cortisol levels between and within studies is significant. This variability partially may be explained by our findings that postoperative drug levels vary despite standardized intraoperative corticosteroid administration. Our data would suggest that low cortisol levels actually may be the result of higher blood levels of corticosteroid rather than underlying adrenal suppression. In this scenario, patients with true underlying adrenal insufficiency and low cortisol levels would be indistinguishable from infants with low cortisol levels secondary to corticosteroid-induced suppression.

Our findings suggest that standard weight-based dosing of corticosteroids may result in the inflammatory/stress response of infants being oversuppressed in some and undersuppressed in others. This leads to the ultimate question that has yet to be answered, "What is optimal suppression of the post-CPB inflammatory/stress response?" The CPB-generated inflammatory/stress response has been well demonstrated, and the younger the age of the child, the more robust the response. ${ }^{9}$ This inflammatory/stress response has been associated with a variety of poor outcomes. Higher levels of the inflammatory markers IL-6 and IL-8 correlate with increased 24-hour lactates and longer ICU lengths of stay for neonates after congenital cardiac surgery. ${ }^{10}$ Similarly, there are some data to suggest that a heightened stress response after cardiac surgery may be maladaptive. Mackie et $\mathrm{al}^{11}$ observed that neonates with higher postoperative cortisol levels had increased atrial filling pressures and a lower cardiac index over the first 48 postoperative hours. Wald et $\mathrm{al}^{12}$ found that patients with larger increases in cortisol after a postoperative cosyntropin stimulation test had higher inotrope and fluid requirements and increased ventilator time and ICU length of stay. Further evidence for the value of corticosteroid suppression of the inflammatory/stress response comes from 3 small randomized clinical trials showing that intraoperative dexamethasone was associated with lower postoperative troponin when compared with placebo. ${ }^{1,5,13}$

In contrast, recent large-scale investigations of the Pediatric Health Information Systems Database have raised concerns that, at least for some patients, the risks of corticosteroids may outweigh the benefits. Two separate analyses using the Pediatric Health Information Systems Database to evaluate pre-CPB corticosteroid use in neonates and 0 to 18 year olds failed to find any clinical benefit. In fact, patients with lower surgical risk according to their RACHS-1 classification, experienced higher rates of infection, insulin use, and longer hospital lengths of stay. ${ }^{14,15}$ These findings, combined with our observation that standard weight-based dosing produces widely variable blood corticosteroid levels requires consideration of a more individualized approach to post-CPB immunomodulation. Patients in RACHS-1 categories 1 through 3 are often older and require shorter $\mathrm{CPB}$ times, which should translate into a less robust inflammatory/stress response. This subset of patients may not require steroids to achieve optimal postoperative recovery. On the other hand, surgical cases in RACHS- 1 categories 4 through 6 are significantly more complex and are associated with a higher risk for morbidity and mortality. Dexamethasone under-responders (ie, the low dexamethasone group) in these categories may experience a suboptimal suppression of their inflammatory/stress response that goes unrecognized using the current weight-based approach to corticosteroid administration.

A third consideration is the possibility that, for some children, intraoperative corticosteroids actually generate an iatrogenic transient adrenal insufficiency. Our study showed that for high dexamethasone responders (ie, those patients with high postoperative dexamethasone levels), cortisol and ACTH levels remained suppressed long after blood levels of dexamethasone had decreased. Dexamethasone levels were near zero within 24 hours of ICU arrival (time 7) and yet cortisol and ACTH levels continued to trend downward for the high dexamethasone group. This time period when the innate stress response remained suppressed but exogenous corticosteroids were no longer being administered could place the patient at increased risk for lability and poor outcome during the acute postoperative period.

The delayed recovery of the cortisol response observed in our study was consistent with the genomic actions of glucocorticoids. Glucocorticoids exert their effects through both genomic and nongenomic pathways. Nongenomic effects are rapid in onset and offset, acting directly at glucocorticoid receptors or through non-receptor-mediated pathways. Genomic effects involve glucocorticoid-receptor binding that initiates downstream alterations in transcription and translation to induce anti-inflammatory cytokine production (eg, IL-10), suppress proinflammatory cytokines (eg, IL-6, IL-8), and impact cortisol release through interactions with the hypothalamic-pituitary-adrenal axis. Genomic mechanisms of action are slower in onset and offset, and likely explain the persistence of low cortisol levels even after dexamethasone is cleared from the blood in the high dexamethasone group. ${ }^{7}$ Whether or not this delayed recovery in the cortisol response creates a clinically significant adrenal insufficiency after pre-CPB dexamethasone administration requires further investigation in a larger population of patients.

Although this was the first study to our knowledge to measure dexamethasone levels after infant $\mathrm{CPB}$, previous investigations have shown a similar variation in drug levels after intraoperative methylprednisolone (MP) administration. ${ }^{8}$ Evaluations of adult glucocorticoid pharmacokinetics and pharmacodynamics have identified a variety of factors that impact drug clearance and bioavailability including age, sex, albumin levels, and chronic hepatic insufficiency. 
The impact of these factors and CPB-related variables on dexamethasone potency and clearance in the infant CHD population are unknown. Metabolism and clearance of dexamethasone is accomplished primarily through the liver. Differences in cytochrome P-450-mediated drug metabolism have been observed within populations of healthy children as well as children with sepsis and multiorgan failure. ${ }^{16,17}$ An increase in the proinflammatory cytokine IL-6 is associated with a reduction in cytochrome P-450-mediated metabolism for a variety of drugs including dexamethasone. ${ }^{16,18}$ Age, co-administration of other cytochrome P-450-metabolized medications, and genetic factors all can impact drug metabolism and clearance. Our study population was too small to evaluate the potential contribution of these characteristics. Ideally, future investigations will seek to identify patientand drug-related characteristics that can facilitate an individualized approach to perioperative corticosteroid administration for infant CPB.

The ultimate challenge is to establish an optimal balance between suppression and augmentation of the inflammatory/stress response. The ideal dexamethasone dose will mediate harmful effects of the inflammatory response, including vasodilation, capillary leak, and cardiac myocyte injury, and simultaneously avoid suppression of adaptive host mechanisms such as cortisol release and immunomodulation that is protective against infection. In our study, inflammatory cytokines, IL-6, and IL-8 levels were very similar between groups, despite significant differences in dexamethasone levels. These findings suggest that low dexamethasone levels $(<15 \mu \mathrm{g} / \mathrm{dL})$ may be sufficient to mediate the inflammatory response without inducing prolonged suppression of the patient's innate stress response. Future investigations must seek to identify patient characteristics that predict maladaptive post-CPB inflammatory responses and impact corticosteroid pharmacokinetics/pharmacodynamics. This knowledge will facilitate an individualized approach that addresses patient-specific CPB response and pharmacodynamics/ pharmacokinetics to inform corticosteroid selection, dose, timing, and duration that will balance the risk/benefit ratio for each patient.

There were several limitations to this study that require further consideration. First, the small sample size in our study precluded rigorous statistical evaluation of all the potential factors that may impact postoperative dexamethasone drug levels. Measurement of these levels in a larger patient population will be required to evaluate the impact of factors such as age, weight, blood loss, transfusion, and patient-specific pharmacokinetics on postoperative corticosteroid drug levels. A second potential limitation was the single-center nature of this investigation. Although the anesthetic, cardiopulmonary bypass, and corticosteroid approach for patients within our center was quite consistent, practice patterns can be highly variable between institutions. We are currently in the process of addressing these limitations with a follow-up study that measures dexamethasone and cortisol levels surrounding infant CPB at 2 independent institutions. Finally, 15 patients in our study received corticosteroids during the postoperative period just before extubation. Patients were censored from the analysis once they received postoperative corticosteroids to ensure that the observed variability in drug levels was not affected by the additional corticosteroid administration. However, these patients were still included in the comparison of the clinical outcomes of mechanical ventilation and ICU length of stay. Despite these limitations, our study was the first to show that postoperative dexamethasone drug levels are highly variable. These findings highlight drug levels as an additional factor that must be considered when evaluating the implications of this highly prevalent clinical practice.

In conclusion, we showed that a standardized $1 \mathrm{mg} / \mathrm{kg}$ dose of dexamethasone given to infants before CPB initiation generates significantly different blood levels of dexamethasone at ICU arrival. These dexamethasone levels are highly correlated with the magnitude of the postoperative cortisol/stress response. Higher dexamethasone levels are associated with suppression of the postoperative cortisol response that persists after dexamethasone blood levels decrease, potentially generating a period of iatrogenic adrenal insufficiency that could impact postoperative stability. These findings establish that corticosteroid blood levels are an important variable to consider when evaluating the role of perioperative corticosteroids for mediating the infant CPB-induced stress/inflammatory response.

\section{References}

1. Heying R, Wehage E, Schumacher K, Tassani P, Haas F, Lange R, et al. Dexamethasone pretreatment provides anti-inflammatory and myocardial protection in neonatal arterial switch operation. Ann Thorac Surg. 2012;93:869-77.

2. Checchia PA, Bronicki RA, Costello JM, Nelson DP. Steroid use before pediatric cardiac operations using cardiopulmonary bypass: an international survey of 36 centers. Pediatr Crit Care Med. 2005;6:441-4

3. Madhok AB, Ojamaa K, Haridas V, Parnell VA, Pahwa S, Chowdhury D. Cytokine response in children undergoing surgery for congenital heart disease. Pediatr Cardiol. 2006;27:408-13.

4. Kozik DJ, Tweddell JS. Characterizing the inflammatory response to cardiopulmonary bypass in children. Ann Thorac Surg. 2006;81:S2347-54.

5. Bronicki RA, Backer CL, Baden HP, Mavroudis C, Crawford SE, Green TP. Dexamethasone reduces the inflammatory response to cardiopulmonary bypass in children. Ann Thorac Surg. 2000;69:1490-5.

6. Suominen PK, Dickerson HA, Moffett BS, Ranta SO, Mott AR, Price JF, et al. Hemodynamic effects of rescue protocol hydrocortisone in neonates with low cardiac output syndrome after cardiac surgery. Pediatr Crit Care Med. 2005;6:655-9.

7. Czock D, Keller F, Maximilian Rasche F, Haussler U. Pharmacokinetics and pharmacodynamics of systemically administered glucocorticoids. Clin Pharmacokinet. 2006;44:61-98.

8. Grosek S, Than A, Wraver B, Gabrijelcic T, Kosin M, Osredkar J, et al. Methylprednisolone, cortisol and the cell-mediated immune response in children after ventricular septal defect repair. Clin Chem Lab Med. 2007;45:1366-72.

9. Alcarez AJ, Manzano L, Sancho L, Vigil MD, Esquivel F, Maroto E, et al. Different proinflammatory cytokine serum pattern in neonate patients undergoing open heart surgery. Relevance of IL-8. J Clin Immunol. 2005;25:238-45. 
10. Allan CK, Newburger JW, McGrath E, Elder J, Psoinos C, Laussen PC, et al. The relationship between inflammatory activation and clinical outcome after infant cardiopulmonary bypass. Anesth Analg. 2010;111:1244-51.

11. Mackie AS, Gauvreau K, Booth KL, Newburger JW, Laussen PC, Roth SJ. Hemodynamic correlates of serum cortisol in neonates after cardiopulmonary bypass. Pediatr Crit Care Med. 2011;12:297-303.

12. Wald EL, Preze E, Eickhoff JC, Backer CL. The effect of cardiopulmonary bypass on the hypothalamic-pituitary-adrenal axis in children. Pediatr Crit Care Med. 2011;12:190-6.

13. Checchia PA, Backer CL, Bronicki RA, Baden HP, Crawford SE, Green TP, et al. Dexamethasone reduces postoperative troponin levels in children undergoing cardiopulmonary bypass. Crit Care Med. 2003;31:1742-5.

14. Pasquali SK, Li JS, He X, Jacobs ML, O’Brien SM, Hall M, et al, Perioperative methylprednisolone and outcome in neonates undergoing heart surgery. Pediatrics. 2012;129:e385-91

15. Pasquali SK, Hall M, Li JS, Peterson ED, Jaggers J, Lodge AJ, et al. Corticosteroids and outcome in children undergoing congenital heart surgery: analysis of the Pediatric Health Information System Database. Circulation. 2010;122:2123-30

16. Carcillo JA, Doughty L, Kofos D, Frye RF, Kaplan SS, Sasser H, et al. Cytochrome p-450 mediated-drug metabolism is reduced in children with sepsis-induced multiple organ failure. Intensive Care Med. 2003;29: 980-4.

17. Ito S, Kusunoki Y, Oka T, Ito Y, Okuno A, Yoshioka H. Pharmacokinetics of high dose methylprednisolone in children. Dev Pharmacol Ther. 1992;19: 99-105.

18. Abdel Razzak Z, Loyer P, Fautrel A, Gautier JC, Corcos L, Turlin B, et al, Cytokines down regulate expression of major cytochrome P450 enzymes in adult human hepatocytes in primary culture. Mol Pharmacol. 1993;44: 707-15.

\section{Discussion}

Dr Olaf Reinhartz (Stanford, Calif). Thank you. Dr Crow, you and your group are to be commended for trying to shed light on a subject that still remains quite nebulous to us. The benefits of perioperative steroids for complex congenital repairs are still unclear, and, as of late, as you mentioned, there is even evidence that steroids might actually be harmful at least in some patients. Practice between institutions varies as widely as one would imagine.

Your study is carried out well and has 3 important findings. Number one, blood drug levels despite consistent drug dosage are highly variable, and this variation is not easily explained by patient factors like age, complexity of the procedure, etcetera. Secondly, expectedly and logically higher drug levels lead to more and longer adrenal suppression. And, third, cytokine levels are not different between high and low drug levels in your 2 groups.

I have 2 questions for you. I will ask them one at a time. The first one relates to the large drug level variability. I cannot come up with an explanation why there would be 2 distinct groups as you are describing them, high versus low dexamethasone levels in your study. Did you just arbitrarily categorize these patients into 2 groups? I did not see a scattergram to facilitate analysis. Or, do you really think there are 2 distinct patient populations and you just have not found out yet what distinguishes them?

Dr Crow. Yes, it is an excellent question. We did not have 2 distinct groups. They were scattered around this $15 \mu \mathrm{g} / \mathrm{dL}$ mark that I chose, and I just tried to find a point that looked like most of the group fell above or below in order to make some comparisons.
There is a wide range of variability, but there does appear to be significant differences between the 2 groups. If you divide on that 15 mark, the groups are significantly different between their levels at each point in time.

We do have some studies in methylprednisolone in adults that demonstrate that there is a large variety of components that could contribute to this. And there have even been some studies by Joe Carcillo that show that even in healthy children, we will have differences in drug metabolism.

So inflammatory markers also play into drug metabolism, and IL-6 specifically has been shown to decrease clearance with the cytochrome P450 system, which is utilized to clear dexamethasone.

We did not find a difference in IL-6 levels in our population that would have explained that, but I think it just highlights that there are many, many questions that still need to be investigated to try to figure out why there is such discrepant levels in these patients.

Dr Reinhartz. Secondly, you and others before you have proposed larger prospective multicenter trials around this topic. How would you design these trials? What should the treatment arms be, particularly in light of the more recent data that you mentioned questioning the benefit of perioperative steroids entirely?

Dr Crow. I love this question because I think it is really where the field needs to go now. I think especially with the latest large studies showing potential negative outcomes of corticosteroid treatment. As a result, the clinical equipoise around the practice has grown even in the last 5 years. I am amazed at how much more willing people are to consider not giving corticosteroids intraoperatively. There are now centers that do not give corticosteroids and centers that do give.

So I do think that while we still have some challenges to get people to buy into a randomized clinical trial, which would be the gold standard, there is a possibility to design a study where we take different centers that have different steroid practices, measure these blood levels in those patients in a larger sample, and try to figure out is the best strategy. Are there drug levels that can suppress the components we need to suppress, and will not suppress things like the immune system that are needed to prevent infection?

We have just completed analysis on samples from an additional center, and so we will have the opportunity to look at patients at 2 different centers and have preliminary data to pursue that kind of multicenter investigation. In this scenario, people could still use their standard practices, but we could monitor how those practices translate into postoperative inflammatory/stress responses and try to draw some conclusions from that information.

Dr James S. Tweddell (Milwaukee, Wis). That was a really very interesting study and an excellent presentation. I have just a couple of questions.

How far in advance of skin incision or initiation of bypass were the steroids given?

Dr Crow. Steroids are usually given immediately after the patients are asleep and then right before the bypass prime.

So we had about an hour time period. I actually went and looked at each of them individually, and for all the patients they got $0.5 \mathrm{mg} / \mathrm{kg}$ of dexamethasone right after induction and then another $0.5 \mathrm{mg} / \mathrm{kg}$ right before bypass was starting. And that was the 
standard protocol for all the patients. This time period was about an hour for each patient.

Dr Tweddell. Did you look at any variability in that interval between the first dose and the second dose? Did that have an impact?

Dr Crow. So we actually did not have very much variability. Outside of about 20 minutes, the intervals between those steroids were very similar. You could also question variability in the time between steroid administration and ICU arrival, since we use the level at ICU arrival to divide the patients into 2 groups. There was variability between when bypass started and when it would have finished, and when the patient arrived to the ICU. In an attempt to control for this variability, we compared bypass times between groups. That is sort of a surrogate marker of how long the operative time is for these patients. There were no significant differences in bypass time between groups.

But, again, this is another area where it would be nice to have a larger patient population where you could look a little more discretely at some of those variables. Twenty minutes might end up being significant if you had enough patients to look at it in.

Dr Tweddell. Is there any potential to go back and look at the ACTH levels in these patients?

Dr Crow. Yes. I can actually show it to you right now.

Dr Tweddell. I'm glad I asked a question you are prepared for.

Dr Crow. Well, I did not have time to put it in. Otherwise, I would have shown you that, too.

But the ACTH levels were quite similar to the cortisol levels, and they were similarly suppressed, as you can see, in that high dexamethasone group.
So, it looks like we suppressed the entire axis. And, in fact, dexamethasone is a more potent suppressor of the stress response than your innate cortisol levels. So when I show that negative feedback loop, our cortisol does not suppress the hypothalamus pituitary as much as dexamethasone does.

So, we are really playing with a pretty powerful mediator. And we know the genomic effects of dexamethasone work through transcription and translation and will actually produce a much more prolonged effect on adrenal suppression even after the blood levels have cleared.

So, I think it is very interesting that we have that time period where we are no longer supplementing. Cortisol levels are low and ACTH levels are low, lower even than when the patient first came to surgery.

I am looking forward to looking closer at that 8- and 24-hour time period and beyond to see if we can actually pick out patients that are more labile during that time or kind of seem to be doing okay and then get worse. Is that part of the problem-that we have created this iatrogenic adrenal insufficiency?

Dr Lyle D. Joyce (Rochester, Minn). Dr Crow, I assume you would be happy to have any centers here that are interested in joining you to contact you, right?

Dr Crow. Yes. That would be great.

Dr Joyce. Because, as you say, there is variability across the country, and it would be nice to sort that out.

Dr Crow. Yes. The key to answering this question will definitely be multicenter evaluation of what we are already doing. 